\title{
Degenerate optical memory effect in dielectric films with randomly rough surfaces
}

\author{
J. A. Sánchez-Gil \\ Instituto de Estructura de la Materia, CSIC, Serrano 121, E-28006 Madrid, Spain
}

(Received 31 January 1997)

\begin{abstract}
By means of perturbation theory we calculate the angular intensity correlations of linearly polarized electromagnetic waves scattered from a thin dielectric film deposited on a planar perfectly conducting substrate. The illuminated surface is a one-dimensional, randomly rough surface with a power spectrum given either by a Gaussian, or by a rectangle defined to enhance the coupling into guided waves. The dielectric film supports, at least, two guided waves in the absence of the roughness that have distinct wave numbers at the frequency $\omega$ of the incident wave. We analyze the angular dependence of the optical memory effect: In addition to its two well known peaks, we demonstrate that, due to degeneracy of the excitation of guided waves, there appear two pairs of peaks symmetrically located about the former absolute maxima at positions related to the difference between the guided wave numbers. This degenerate memory effect is shown to be a multiple scattering effect. [S0163-1829(97)04723-1]
\end{abstract}

\section{INTRODUCTION}

The scattering of electromagnetic waves from dense random media and rough surfaces has been intensively investigated in recent years. ${ }^{1,2}$ A great deal of attention has been paid not only to the first statistical moments of the scattered intensity, such as the angular distribution of its average, but also to other relevant physical magnitudes such as the intensity correlations. These correlations describe how the speckle pattern, formed as a result of the interference among randomly scattered waves, evolves when one or more parameters of the scattering system are changed. In early work, Leger $e t a l .^{3}$ studied both experimentally and theoretically the angular intensity correlations, finding that the speckle pattern rotates as the direction of the incident beam is changed, simultaneously losing its resemblance with the initial intensity distribution. With respect to this, we mention the work by Goodman ${ }^{4}$ on this and other statistical properties of the scattered speckle patterns.

Later on, Feng et al..$^{5}$ have calculated angular correlation effects of both short and long range in scalar waves transmitted through a disordered medium. Similar to the phenomenon addressed in the works quoted above for single scattering, ${ }^{3,4}$ the short-range correlation, called memory effect (ME), predicts the existence of maxima in the angular correlation of the speckle patterns when the difference between transverse incident wave vectors coincides with the difference between transverse scattered wave vectors provided that this difference is not too large. The width of this correlation is, however, considerably smaller for multiply scattered waves than it is for single scattered waves. This effect, also found for scalar waves in reflection, has been experimentally confirmed, ${ }^{6}$ and theoretically corroborated by means of a real-space approach, ${ }^{7}$ also predicting a timereversed analog ${ }^{8}$ as a consequence of the reciprocity principle. The influence of the vectorial nature of the electromagnetic waves on the ME has been subsequently taken into account. ${ }^{9}$ On the other hand, with regard to random systems with surface disorder, the angular intensity correlation has been studied by means of rigorous numerical simulation cal- culations of the electromagnetic waves scattered from rough surfaces for which there exist strong double scattering contributions, accounting for the ME (Refs. 10 and 11) and other enhanced long-range correlations. ${ }^{11,12}$ Incidentally, a similar ME has been reported in the double passage of waves through a random phase screen. ${ }^{13}$

Most of the above mentioned works deal with infinite or semi-infinite systems, or with finite systems for which the spatial confinement does not play a relevant role in wave propagation. Very recently, Freilikher et al. ${ }^{14}$ predicted in the case of a disordered, bounded system with a discrete spectrum of eigenmodes the appearance in the mean scattered intensity of satellite peaks in addition to the well known backscattering peak. The existence of such peaks has also been shown by perturbation theories and numerical simulations of the light scattered from bounded systems with surface disorder, such as thin dielectric or metallic films with a randomly rough surface (cf. Refs. 15-17).

It is our aim in this paper to study theoretically the influence of degenerate time reversal symmetry in the ME of the $s$ - and $p$-polarized waves scattered from one of such bounded systems with surface disorder. Particularly, we consider a one-dimensionally rough dielectric film deposited on a planar, perfectly conducting substrate. To calculate the angular intensity correlations, we develop a small amplitude perturbation theory of the field correlation function valid through terms of fourth order in the surface profile function, thus taking into account double scattering processes. (The factorization approximation, valid for short-range correlations, is applied in order to relate the intensity correlation to the field correlation.) This perturbation theory will give physical insight into the effects we are interested in through the explicit analytic expressions it provides. Furthermore, it has proven successful in describing quantitatively the mean scattered intensity of electromagnetic waves from a rough dielectric film similar to the one being studied here. ${ }^{18}$ Interestingly, with respect to the statistical properties of the random surface, it should be mentioned that two power spectra have been analyzed. On the one hand, a Gaussian power spectrum with a small correlation length that resembles 


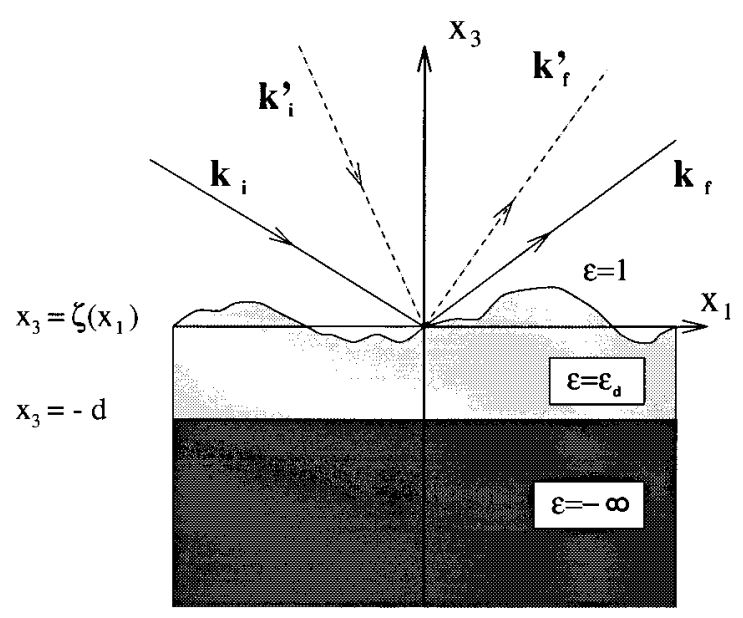

FIG. 1. The rough dielectric film on a perfectly conducting substrate studied in the present paper.

many naturally occurring surface roughnesses and is widely used in experiments; on the other hand, a rectangular power spectrum especially designed so that maximum light coupling into guided waves takes place for a certain range of incidence angles near the normal direction. The latter power spectrum has been experimentally employed by West and O'Donnell in studies of enhanced backscattering from rough metal surfaces to maximize the excitation of surface-plasmon polaritons ${ }^{19}$ (cf. Ref. 20 for subsequent theoretical work).

The outline of this paper is the following. In Sec. II, we describe the dielectric film with a one-dimensional, randomly rough surface studied in this work, and the angular dependence of the intensity correlation of linearly (either $s$ or $p$ ) polarized electromagnetic waves scattered from the rough film is calculated perturbatively. The results for the degenerate ME obtained by the use of this perturbation theory are shown and discussed in Sec. III. The main conclusions drawn from the results of our calculations are summarized in Sec. IV. An Appendix in which certain results needed in the perturbation theory are collected concludes this paper.

\section{INTENSITY CORRELATION: PERTURBATION THEORY}

Let us consider a dielectric film of mean thickness $d$ deposited on a planar, perfectly conducting substrate occupying the region $x_{3}<-d$ (Fig. 1). The vacuum-dielectric interface is a one-dimensional randomly rough surface, whose surface profile function $\zeta\left(x_{1}\right)$ is a continuous, single-valued function of $x_{1}$, that is differentiable as many times as is necessary. In addition, $\zeta\left(x_{1}\right)$ is assumed to be a stationary, Gaussian stochastic process of zero mean. The rms height of the surface is $\delta=\left\langle\zeta^{2}\left(x_{1}\right)\right\rangle^{1 / 2}$, where the angle brackets denote an average over the ensemble of realizations of $\zeta\left(x_{1}\right)$. The Fourier coefficient $\hat{\zeta}(k)$,

$$
\hat{\zeta}(k)=\int_{-\infty}^{\infty} d x_{1} \zeta\left(x_{1}\right) \exp \left(-i k x_{1}\right)
$$

is also a Gaussian-random process of zero mean that satisfies the following statistical property:

$$
\left\langle\hat{\zeta}(k) \hat{\zeta}\left(k^{\prime}\right)\right\rangle=2 \pi \delta\left(k+k^{\prime}\right) \delta^{2} g(|k|),
$$

$g(|k|)$ being the power spectrum of the surface roughness.

We aim to study the angular intensity correlation function defined as

$$
C\left(\mathbf{k}_{i}, \mathbf{k}_{f} ; \mathbf{k}_{i}^{\prime}, \mathbf{k}_{f}^{\prime}\right)=\frac{\left\langle\Delta I\left(\mathbf{k}_{i}, \mathbf{k}_{f}\right) \Delta I\left(\mathbf{k}_{i}^{\prime}, \mathbf{k}_{f}^{\prime}\right)\right\rangle}{\left\langle I\left(\mathbf{k}_{i}, \mathbf{k}_{f}\right)\right\rangle\left\langle I\left(\mathbf{k}_{i}^{\prime}, \mathbf{k}_{f}^{\prime}\right)\right\rangle},
$$

with $\Delta I=I-\langle I\rangle$. This function correlates the speckle intensity appearing at the scattering direction $\mathbf{k}_{f}^{\prime}$, upon impinging on the rough film at the incidence direction $\mathbf{k}_{i}^{\prime}$, with that speckle produced at the scattering direction $\mathbf{k}_{f}$ when moving the incidence to $\mathbf{k}_{i}$ (cf. Fig. 1).

In what follows, we restrict our analysis to $s$ - and $p$-polarized electromagnetic waves of frequency $\omega$, whose plane of incidence is perpendicular to the generators of the rough surface. Thus no depolarization takes place. Furthermore, the present scattering geometry makes it easier to simplify the formulation by dealing with the only nonzero component of either the electric vector for $s$ polarization,

$$
\mathbf{E}(\mathbf{x} ; t)=\left[0, E_{2}\left(x_{1}, x_{3} \mid \omega\right), 0\right] \exp (-i \omega t),
$$

or the magnetic vector for $p$ polarization,

$$
\mathbf{H}(\mathbf{x} ; t)=\left[0, H_{2}\left(x_{1}, x_{3} \mid \omega\right), 0\right] \exp (-i \omega t) .
$$

The field amplitude in the vacuum region $x_{3}>\zeta\left(x_{1}\right)$ can be written as the sum of the incident beam (a plane wave in this case) and the scattered field

$$
\begin{aligned}
F_{\gamma}^{>}\left(x_{1}, x_{3} \mid \omega\right)= & \exp \left[i k x_{1}-i \alpha_{0}(k, \omega) x_{3}\right] \\
& +\int_{-\infty}^{\infty} \frac{d p}{2 \pi} R_{\gamma}(p \mid k) \exp \left[i p x_{1}+i \alpha_{0}(p, \omega) x_{3}\right]
\end{aligned}
$$

where $\gamma=s, p$ denotes the polarization state and $F_{s}^{>}=E_{2}^{>}\left(x_{1}, x_{3} \mid \omega\right), F_{p}^{>}=H_{2}^{>}\left(x_{1}, x_{3} \mid \omega\right)$. In Eq. (2.6),

$$
\begin{aligned}
\alpha_{0}(p, \omega) & =\left[\left(\omega^{2} / c^{2}\right)-p^{2}\right]^{1 / 2} \quad p^{2}<\left(\omega^{2} / c^{2}\right) \\
& =i\left[p^{2}-\left(\omega^{2} / c^{2}\right)\right]^{1 / 2} \quad p^{2}>\left(\omega^{2} / c^{2}\right) .
\end{aligned}
$$

$R_{\gamma}(p \mid k)$ is the scattering amplitude of a plane wave incident with wave vector $\mathbf{k}_{i} \equiv\left[k, 0, \alpha_{0}(k, \omega)\right]$ [and angle of incidence $\theta_{i}$ such that $\left.k=(\omega / c) \sin \theta_{i}\right]$ into the plane wave component with wave vector $\mathbf{k}_{f} \equiv\left[p, 0, \alpha_{0}(p, \omega)\right]$ [and angle of scattering $\theta_{s}$ such that $p=(\omega / c) \sin \theta_{s}$, provided that $\left.|p|<\omega / c\right]$. Expressions similar to Eq. (2.6) can be obtained for the field amplitude inside the dielectric film $F_{\gamma}^{<}$.

In order to calculate the scattering amplitudes, the boundary conditions at the interfaces $x_{3}=-d$ and $x_{3}=\zeta\left(x_{1}\right)$ are imposed onto the field amplitudes in the vacuum half space and within the dielectric film. (Note that upon doing so, we are invoking the Rayleigh hypothesis.) It has been shown in Refs. 15 and 17 that the resulting coupled integral equations can be decoupled to yield a single integral equation (reduced Rayleigh equation) for the scattering amplitude in the vacuum half-space for each polarization $R_{\gamma}(p \mid k)$. We proceed now by postulating that this scattering amplitude obeys 
the following expression, in accordance with the many-body perturbation theory formalism: ${ }^{21}$

$$
\begin{aligned}
R_{\gamma}(p \mid k)= & 2 \pi \delta(p-k) R_{\gamma}^{(0)}(k, \omega) \\
& -2 i G_{\gamma}^{(0)}(p, \omega) U_{\gamma}(p \mid k) G_{\gamma}^{(0)}(k, \omega) \alpha_{0}(k, \omega) .
\end{aligned}
$$

$R_{\gamma}^{(0)}(k, \omega)$ is the Fresnel coefficient for the scattering of $\gamma$-polarized light from a dielectric film deposited on a perfectly conducting substrate, when the vacuum/dielectric and dielectric/substrate interfaces are planar and parallel. Namely,

$$
R_{\gamma}^{(0)}(k, \omega)=\frac{D_{\gamma}^{(-)}(k, \omega)}{D_{\gamma}^{(+)}(k, \omega)}
$$

with

$$
\begin{aligned}
D_{s}^{( \pm)}(k, \omega)= & \pm i \alpha_{d}(k, \omega) \cos \alpha_{d}(k, \omega) d \\
& +\alpha_{0}(k, \omega) \sin \alpha_{d}(k, \omega) d, \\
D_{p}^{( \pm)}(k, \omega)= & i \epsilon_{d} \alpha_{0}(k, \omega) \cos \alpha_{d}(k, \omega) d \\
& \pm \alpha_{d}(k, \omega) \sin \alpha_{d}(k, \omega) d,
\end{aligned}
$$

where

$$
\begin{aligned}
\alpha_{d}(k, \omega) & =\left[\epsilon_{d}\left(\omega^{2} / c^{2}\right)-k^{2}\right]^{1 / 2}, \quad k^{2}<\epsilon_{d}\left(\omega^{2} / c^{2}\right) \\
& =i\left[k^{2}-\epsilon_{d}\left(\omega^{2} / c^{2}\right)\right]^{1 / 2}, \quad k^{2}>\epsilon_{d}\left(\omega^{2} / c^{2}\right) .
\end{aligned}
$$

The function $G_{\gamma}^{(0)}(k, \omega)$ is a Green's function for the same dielectric film on a perfectly conducting substrate system:

$$
\begin{gathered}
G_{s}^{(0)}(k, \omega)=\frac{i \sin \alpha_{d}(k, \omega) d}{D_{s}^{(+)}(k, \omega)} ; \\
G_{p}^{(0)}(k, \omega)=\frac{-\epsilon_{d} \cos \alpha_{d}(k, \omega) d}{D_{p}^{(+)}(k, \omega)} .
\end{gathered}
$$

Furthermore, before going on to the calculation of the transition matrix $U_{\gamma}(p \mid k)$ itself, this transition matrix is postulated to satisfy the equations

$$
\begin{aligned}
U_{\gamma}(p \mid k) & =V_{\gamma}(p \mid k)+\int_{-\infty}^{\infty} \frac{d q}{2 \pi} V_{\gamma}(p \mid q) G_{\gamma}^{(0)}(q, \omega) U_{\gamma}(q \mid k) \\
& =V_{\gamma}(p \mid k)+\int_{-\infty}^{\infty} \frac{d q}{2 \pi} U_{\gamma}(p \mid q) G_{\gamma}^{(0)}(q, \omega) V_{\gamma}(q \mid k),
\end{aligned}
$$

where $V_{\gamma}(p \mid k)$ is the reflection potential. This reflection potential is related to the scattering amplitude through Eqs. (2.8) and (2.13), and thus can be shown to satisfy an integral equation [cf. Eq. (A12) in Ref. 15 for $s$ polarization, and Eq. (34) in Ref. 17 for $p$ polarization], containing the information provided by the boundary conditions. These integral equations constitute the basis of our perturbation theory. We seek $V_{\gamma}(p \mid k)$ as an expansion in powers of the surfaceprofile function $\zeta\left(x_{1}\right)$,

$$
V_{\gamma}(p \mid k)=\sum_{n=1}^{\infty} V_{\gamma}^{(n)}(p \mid k)
$$

where the superscript denotes the order of the corresponding term in $\zeta\left(x_{1}\right)$. For our purpose, it suffices to consider terms of $V_{\gamma}(p \mid k)$ through third order, which can be written in the form:

$$
V_{\gamma}^{(1)}(p \mid k)=v_{\gamma}^{(1)}(p \mid k) \hat{\zeta}^{(1)}(p-k)
$$

$$
V_{\gamma}^{(2)}(p \mid k)=\int_{-\infty}^{\infty} \frac{d q}{2 \pi} v_{\gamma}^{(2)}(p|q| k)
$$

$$
\times \hat{\zeta}^{(1)}(p-q) \hat{\zeta}^{(1)}(q-k),
$$

$$
\begin{aligned}
V_{\gamma}^{(3)}(p \mid k)= & \int_{-\infty}^{\infty} \frac{d q}{2 \pi} \int_{-\infty}^{\infty} \frac{d r}{2 \pi} v_{\gamma}^{(3)}(p|q| r \mid k) \hat{\zeta}^{(1)}(p-q) \\
& \times \hat{\zeta}^{(1)}(q-r) \hat{\zeta}^{(1)}(r-k)
\end{aligned}
$$

where

$$
\hat{\zeta}^{(n)}(Q)=\int_{-\infty}^{\infty} d x_{1} e^{-i Q x_{1}} \zeta^{n}\left(x_{1}\right)
$$

The functions $v_{\gamma}^{(1)}(p \mid k), v_{\gamma}^{(2)}(p|q| k)$, and $v_{\gamma}^{(3)}(p|q| r \mid k)$ have been obtained in previous perturbation-theoretic calculations of the mean scattered intensity [cf. Eqs. (A14) in Ref. 15 for $s$ polarization, and Eqs. (A1)-(A4) in Ref. 17 for $p$ polarization].

Let us write the correlation function (2.3) in terms of the transition matrix $U_{\gamma}(p \mid k)$. For that purpose, the factorization approximation

$$
\left\langle\Delta I \Delta I^{\prime}\right\rangle=\left|\left\langle R_{\gamma} R_{\gamma}^{\prime *}\right\rangle\right|^{2}
$$

is used. Consequently, with the aid of Eqs. (2.8) and (2.17), the intensity-correlation function is given by

$$
\begin{aligned}
C_{\gamma}\left(p, k ; p^{\prime}, k^{\prime}\right) & =\left|C_{E, \gamma}\left(p, k ; p^{\prime}, k^{\prime}\right)\right|^{2} \\
& =\frac{\left|\left\langle U_{\gamma}(p \mid k) U_{\gamma}^{*}\left(p^{\prime} \mid k^{\prime}\right)\right\rangle+\delta(p-k) \delta\left(p^{\prime}-k^{\prime}\right) B_{\gamma}\left(k, k^{\prime}\right)\right|^{2}}{\left\langle\left|U_{\gamma}(p \mid k)+\delta(p-k) A_{\gamma}(k)\right|^{2}\right\rangle\left\langle\left|U_{\gamma}\left(p^{\prime} \mid k^{\prime}\right)+\delta\left(p^{\prime}-k^{\prime}\right) A_{\gamma}\left(k^{\prime}\right)\right|^{2}\right\rangle} .
\end{aligned}
$$


The particular form of the functions $A_{\gamma}(k)$ and $B_{\gamma}\left(k, k^{\prime}\right)$ will not be shown [it can be straightforwardly calculated from Eqs. (2.3) and (2.8)], for we will not consider the correlation between speckle patterns at specular directions. As a matter of fact, for the weakly rough film surfaces dealt with in this work (for which the perturbation method indeed holds), the specular component of the scattered intensity should be much larger than the diffuse component. Under these circumstances, the specular speckle patterns become partially developed, leading to strong correlations and low speckle contrast, which have been extensively studied, ${ }^{22,23}$ and lie out of the scope of the present work. On the other hand, in Eq. (2.18) we have redefined the intensity correlation func- tion as the square modulus of the amplitude correlation function $C_{E, \gamma}\left(p, k ; p^{\prime}, k^{\prime}\right)$ (this is precisely the factorization approximation mentioned above). Strictly speaking, our perturbation theory is based on the expansion of $C_{E}$ in powers of the rough surface height, rather than in the expansion of $C$ itself. By employing Eqs. (2.13)-(2.15) in Eq. (2.19), and keeping terms in $C_{E}$ up to fourth order in $\zeta\left(x_{1}\right)$, we are led to

$$
C_{\gamma}\left(p, k ; p^{\prime}, k^{\prime}\right)=C_{\gamma}^{\mathrm{ME}, \mathrm{diff}}\left(p, k ; p^{\prime}, k^{\prime}\right)+C_{\gamma}^{\mathrm{ME}, \mathrm{spec}}\left(p, k ; p^{\prime}, k^{\prime}\right),
$$

with $C_{\gamma}^{\mathrm{ME}, \mathrm{spec}} \propto \delta(p-k) \delta\left(p^{\prime}-k^{\prime}\right)$ accounting for all terms at specular directions (thus omitted here), and

$$
\begin{aligned}
C_{\gamma}^{\mathrm{ME}, \mathrm{diff}}\left(p, k ; p^{\prime}, k^{\prime}\right)= & \delta\left(p-k-\left(p^{\prime}-k^{\prime}\right)\right) \mid C_{E, \gamma}^{(1-1)}\left(p, k ; p^{\prime}, k^{\prime}\right)+C_{E, \gamma}^{(2-2) L}\left(p, k ; p^{\prime}, k^{\prime}\right)+C_{E, \gamma}^{(2-2) C}\left(p, k ; p^{\prime}, k^{\prime}\right) \\
& +\left.C_{E, \gamma}^{(1-3)}\left(p, k ; p^{\prime}, k^{\prime}\right)\right|^{2} .
\end{aligned}
$$

Equation (2.21) constitutes the main result of this work, and describes the optical memory effect at nonspecular angles by means of a perturbation theory of the amplitude correlation function up to fourth order in the surface profile function. The corresponding terms in the rhs of Eq. (2.21) are given in the Appendix as a function of the first three terms of the expansion of the scattering potential in powers of $\zeta\left(x_{1}\right)$ [cf. Eqs. (2.15)], and the Green's function $G_{\gamma}^{(0)}(k, \omega)$. In Eqs. (2.21), $C_{E}^{(1-1)}$ yields the second-order contribution, and $C_{E, \gamma}^{(2-2) L}, C_{E, \gamma}^{(2-2) C}$, and $C_{E, \gamma}^{(1-3)}$ give the contributions of fourth order in $\zeta\left(x_{1}\right)$ from, respectively, the ladder term, the maximally crossed term, and the contribution arising from the products of first- and third-order terms in $U_{\gamma}$. In this regard, it should be mentioned that the intensity correlation function (2.21) is completely general and could be applied (within the validity of the perturbation method) to any other scattering system provided that the appropriate Green's function and scattering potential are taken into account.

\section{RESULTS AND DISCUSSION}

We now present results of numerical calculations of intensity angular correlations on the basis of the perturbationtheoretic analysis described above which has led to Eqs. (2.20) and (2.21). In particular, we choose a dielectric film of mean thickness $d=500 \mathrm{~nm}$ whose dielectric constant is $\epsilon_{d}=2.6896+\imath 0.0075$, deposited on a perfectly conducting substrate. The wavelength of the incident plane wave is $\lambda=632.8 \mathrm{~nm}$. The upper vacuum-dielectric interface is a one-dimensional random surface [cf. Sec. II] with rms height $\delta=15 \mathrm{~nm}$. Two kinds of rough surfaces are considered according to their power spectrum $g(|k|)$ [cf. Eq. (2.2)]: A Gaussian power spectrum (GPS)

$$
g(|k|)=\pi^{1 / 2} a \exp \left(-k^{2} a^{2} / 4\right),
$$

with transverse correlation length $a=100 \mathrm{~nm}$; and a rectangular power spectrum (RPS)

$$
\begin{aligned}
g(|k|) & =\pi\left(k_{\max }-k_{\min }\right)^{-1}, \quad|k| \in\left[k_{\min }, k_{\max }\right] \\
& =0, \quad|k| \notin\left[k_{\min }, k_{\max }\right] .
\end{aligned}
$$

The RPS has been proposed, and employed experimentally, by West and O'Donnell ${ }^{19}$ (cf. also the subsequent theoretical work by Maradudin et al. ${ }^{20}$ ) in the case of rough metal surfaces to enhance the roughness-induced coupling of EM waves into surface plasmon polaritons for an adequate choice of the parameters $k_{\min }$ and $k_{\max }$ (which depends on the angle of incidence and the wave vector of the surface plasmon polariton ${ }^{19,20}$ ). These parameters are chosen in our case with the aim of enhancing the excitation of GW polaritons through the surface roughness. For the values of $\epsilon_{d}$, $d$, and $\lambda$ given above, it has been shown in Ref. 15 from the corresponding dispersion relation that the dielectric waveguide in the absence of roughness supports two $s$-polarized (TE) GWs with wave vectors $q_{1}^{(s)}=(\omega / c) 1.547$ and $q_{2}^{(s)}=(\omega / c) 1.242$. Upon assuming that these values are not significantly perturbed by the presence of roughness, ${ }^{15}$ the excitation of both forward and backward propagating $q_{1}^{(s)}$ and $q_{2}^{(s)}$ is ensured with

$$
\begin{aligned}
& k_{\text {min }}=q_{2}^{(s)}-\frac{\omega}{c} \sin 10^{\circ}=\frac{\omega}{c} 1.0687, \\
& k_{\text {max }}=q_{1}^{(s)}+\frac{\omega}{c} \sin 10^{\circ}=\frac{\omega}{c} 1.7202,
\end{aligned}
$$

for an angle of incidence $\left|\theta_{0}\right| \leqslant 10^{\circ}$.

Before presenting the perturbation-theoretic results, it should be remarked that for these choices for the values of $\delta, \lambda, a$, and $k_{\min }$ and $k_{\max }$, the conditions of both small rms height (small parameter in the perturbative expansion of the scattering potentials) and small rms slope (validity of the Rayleigh hypothesis) are well satisfied. Moreover, the validity of the perturbation-theoretic calculation of the mean scattered intensity has been quantitatively proved for a random surface with a GPS and similar parameters to those consid- 


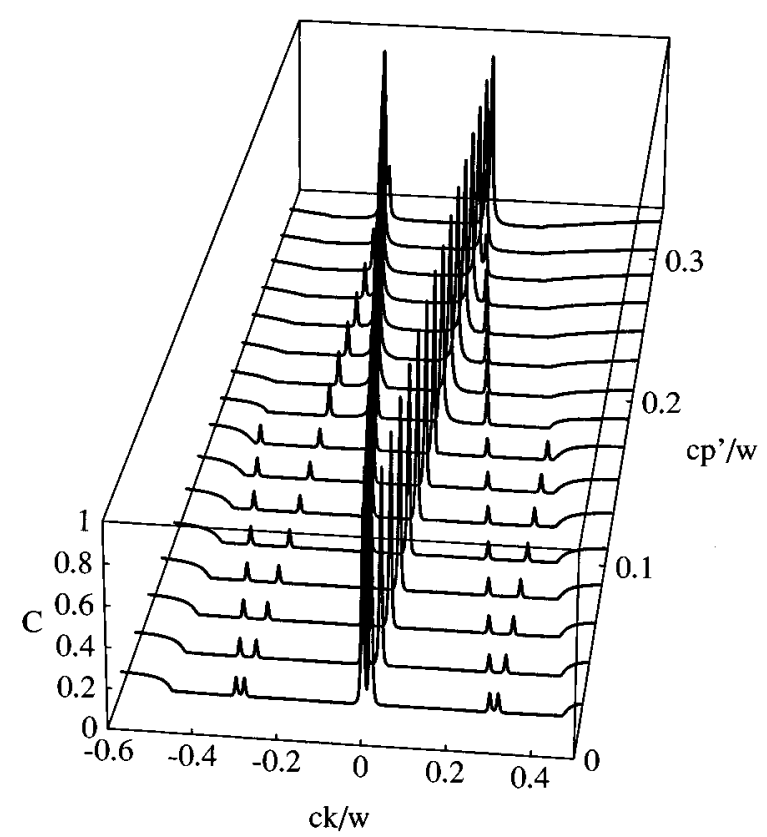

FIG. 2. Contribution through fourth order in the surface profile function to the angular intensity correlation for $s$-polarized electromagnetic waves of wavelength $\lambda=632.8 \mathrm{~nm}$ incident on a dielectric film of mean thickness $d=500 \mathrm{~nm}$ lying on a perfectly conducting substrate. The dielectric constant at that wavelength is $\epsilon=2.6896+i 0.0075$. The one-dimensional, randomly rough, vacuum-dielectric interface is characterized by the rms height $\delta=15 \mathrm{~nm}$ and the rectangular power spectrum defined by Eqs. (3.2) and (3.3). The reference direction of incidence is $\mathrm{k}^{\prime}=0$; the variation of the memory effect envelope $\left(p-k=p^{\prime}-k^{\prime}\right)$ vs $k$ is shown for different reference scattering directions $p^{\prime}$.

ered here by direct comparisons with rigorous numerical simulation calculations, revealing an excellent agreement. ${ }^{18}$

The angular-correlation function $C_{\gamma}\left(p, k ; p^{\prime}, k^{\prime}\right)$ given by Eq. (2.20) depends on four parameters $p, k, p^{\prime}$, and $k^{\prime}$, but the ME condition expressed by the Dirac $\delta$ function in Eq. (2.21) constrains the number of independent parameters to three. In all the calculations presented below, the reference angle of incidence is $0^{\circ}$ (namely, $k^{\prime}=0$ ), and the $2 \pi / L$ factor arising from the $\delta$ function is taken into account. Typically, for a fixed value of the reference scattering direction $p^{\prime}$, we scan across the incident direction $k$ of the second speckle pattern being correlated with the reference one; the ME condition mentioned above then requires that $p=k+p^{\prime}-k^{\prime}$. In Fig. 2, the results for the angular-intensity correlation are shown in the case of $s$ polarization and a rough surface with the RPS [cf. Eqs. (3.2) and (3.3)]. Several scans in $c k / \omega$ (ME envelopes) are plotted for different values of $c p^{\prime} / \omega$ ranging from 0.02 to 0.4 . Various peaks appear in Fig. 2. On the one hand, two absolute maxima $(C=1)$ are seen that obey the following conditions:

$$
\begin{gathered}
k=k^{\prime}, \\
k=-p^{\prime},
\end{gathered}
$$

which reveal the total correlation existing between, respectively, identical speckle patterns [Eq. (3.4a)], and those speckle patterns related by time-reversal symmetry $[\mathrm{Eq}$. (3.4b)]. In addition, two pairs of small peaks can be observed in Fig. 2. These pairs consist of two local maxima symmetrically placed about the two large peaks [cf. Eq. (3.4)], and follow the displacement of the latter peaks satisfying the conditions

$$
\begin{gathered}
k=k^{\prime} \pm q_{1}-q_{2}, \\
k=-p^{\prime} \pm q_{1}-q_{2}
\end{gathered}
$$

with $q_{1}^{(s)}-q_{2}^{(s)}=(\omega / c) 0.305$, as mentioned above (recall also that $k^{\prime}=0$ in Fig. 2). These peaks manifest the fact that a higher correlation in the memory effect is expected between speckle patterns stemming from multiple scattering trajectories mediated by two degenerate GWs when the phase difference between these trajectories vanishes. Since the two GWs have distinct wave vectors, local maxima are found for different incident and scattering directions of the correlated speckle patterns; these directions are in turn related to the difference between the guided wave vectors through Eq. (3.5a) [condition (3.5b) arises from timereversal symmetry]. This phenomenon is called degenerate optical memory effect. It is analogous in the intensity correlation function to the satellite peaks observed in the mean scattered intensity, ${ }^{15,17}$ which, in fact, satisfy Eq. (3.5b) with $p^{\prime}=p$. Moreover, the angular width of the degenerate ME peaks (evaluated in the amplitude correlation function) is similar to that of the satellite peaks, ${ }^{15}$ and is indeed related to the inverse of the GW mean free path. A similar correspondence, involving the backscattering and ME peaks for random metal surfaces, has been explicitly studied. ${ }^{24}$

In light of the preceding discussion, and upon recalling the precise form of the RPS of the rough surface [cf. Eqs. (3.2) and (3.3)], it is also easy to understand why the degenerate $\mathrm{ME}$ peaks corresponding to both the $(+)$ sign in Eq. (3.5a) and the ( - ) sign in Eq. (3.5b) disappear in Fig. 2 for $p^{\prime}>(\omega / c) \sin 10^{\circ}$. For these values of $p^{\prime}$, it turns out that $k<-(\omega / c) \sin 10^{\circ}-\left(q_{1}^{(s)}-q_{2}^{(s)}\right)=k_{\min }-q_{1}^{(s)}=-k_{\max }+q_{2}^{(s)}$ for the $(-)$ peak in Eq. (3.5b), which makes it unfeasible to couple the incident light into either GW. In contrast, such coupling is possible for the $(+)$ peak in Eq. (3.5a) for $p^{\prime}>(\omega / c) \sin 10^{\circ}$, but the back conversion of the thus excited GWs into scattered light is forbidden for the required scattering directions $p=k+\left(p^{\prime}-k^{\prime}\right)>(\omega / c) \sin 10^{\circ}$ $+\left(q_{1}^{(s)}-q_{2}^{(s)}\right)=k_{\max }-q_{2}^{(s)}=-k_{\min }+q_{1}^{(s)}$. Even though not all the possible double-scattering trajectories involving the excitation of the two supported GWs are allowed by the RPS of the surface roughness, it can be shown that an appropriate combination of these trajectories exists for the two speckle patterns being correlated that gives rise to the remaining peaks appearing in Fig. 2.

To gain more insight into the physical origin of the degenerate memory effect, the different contributions to the intensity angular correlation of Fig. 2 can be plotted separately. This is done in Fig. 3 as a function of $\theta_{0}=\sin ^{-1}(c k / \omega)$ for $p^{\prime}=(\omega / c) 0.1$. Note that the contributions to Eq. (2.21) arising from both the second-order term $C_{E, s}^{(1-1)}$, Eq. (A1), and from the fourth-order term $C_{E, s}^{(1-3)}$, Eq. (A4), vanish for the range of incident and scattering directions considered in Fig. 3 (and also for that of Fig. 2). Therefore, only those contributions coming from the ladder 


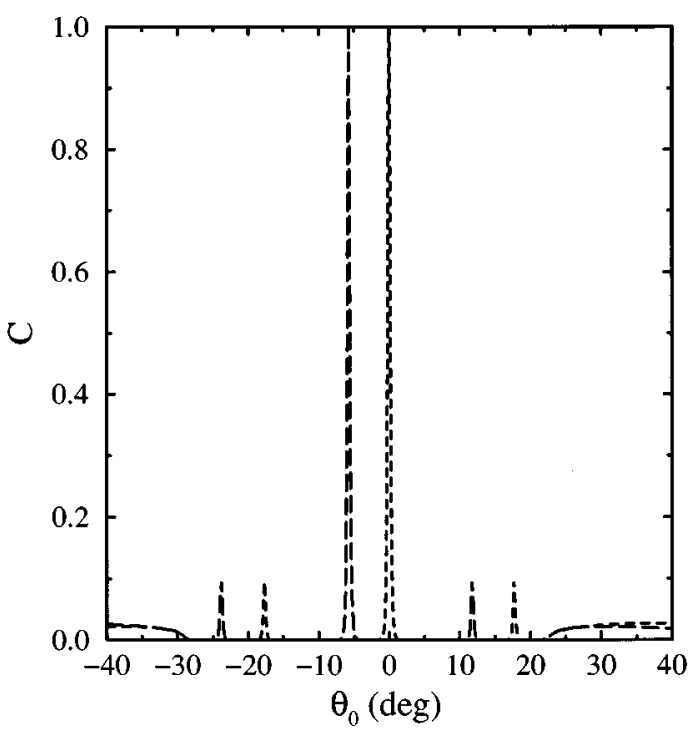

FIG. 3. Same as Fig. 2 for $c p^{\prime} / \omega=0.1$, but including only the fourth-order contributions to the ME envelope arising from the ladder (dashed curve) and crossed (long-dashed curve) terms. Both the second-order contribution (1-1) and the fourth-order contribution from the (1-3) term vanish. The incident angle along the $x$ axis is $\theta_{0}=\sin ^{-1}(c k / \omega)$.

$C_{E, s}^{(2-2) L}$, Eq. (A2), and crossed $C_{E, s}^{(2-2) C}$, Eq. (A3), components of fourth order are shown in Fig. 3. As expected, the self-correlation ME maximum [cf. Eq. (3.4a)] and its degenerate satellite maxima [cf. Eq. (3.5a)] are obtained in the ladder contribution. In contrast, the time-reversed ME maximum [cf. Eq. (3.4b)] and its corresponding degenerate satellite maxima [cf. Eq. (3.5b)] appear only in the maximally crossed contribution, for this contribution accounts, indeed, for the interference effects between a doubly scattered trajectory of one speckle and the time-reversed partner of another trajectory corresponding to the second speckle pattern being correlated.

In Fig. 4, the perturbation-theoretic results for the intensity angular correlation as a function of both $p^{\prime}$ and $k$ $\left(k^{\prime}=0\right.$ and $\left.p=k+p^{\prime}-k^{\prime}\right)$ are shown as in Fig. 2 but in the case of $p$ polarization. The rough surface has a RPS as described in Eqs. (3.2) and (3.3). As in the case of $s$ polarization, the strong $C=1$ maxima are seen at the expected positions Eq. (3.4). Nevertheless, four degenerate-ME maxima, rather than two, appear symmetrically placed about the former absolute maxima in Fig. 4. This can be understood by looking at the dispersion relation of the $p$-polarized (TM) GWs supported by the dielectric film in the absence of roughness. From the corresponding equation [cf. Eq. (12) in Ref. 17], three TM GWs are found for the values of $\epsilon_{d}, d$, and $\lambda$ given above: $q_{1}^{(p)}=(\omega / c) 1.612, q_{2}^{(p)}=(\omega / c) 1.382$, and $q_{3}^{(p)}=(\omega / c) 1.003$. Thus one would expect degenerate ME maxima at the directions determined by Eqs. (3.5a) and (3.5b), but with the wave vectors $q_{n}$ being any combination of two of the former three GW polaritons: $\Delta q_{12}^{(p)}=q_{1}^{(p)}-q_{2}^{(p)}=(\omega / c) 0.23, \quad \Delta q_{23}^{(p)}=(\omega / c) 0.379, \quad$ and $\Delta q_{13}^{(p)}=(\omega / c) 0.609$. Whereas the latter yields peaks outside the range of Fig. 4 [or forbidden for the required GW excitation, as is the case of the (+) solution to Eq. (3.5b)], the

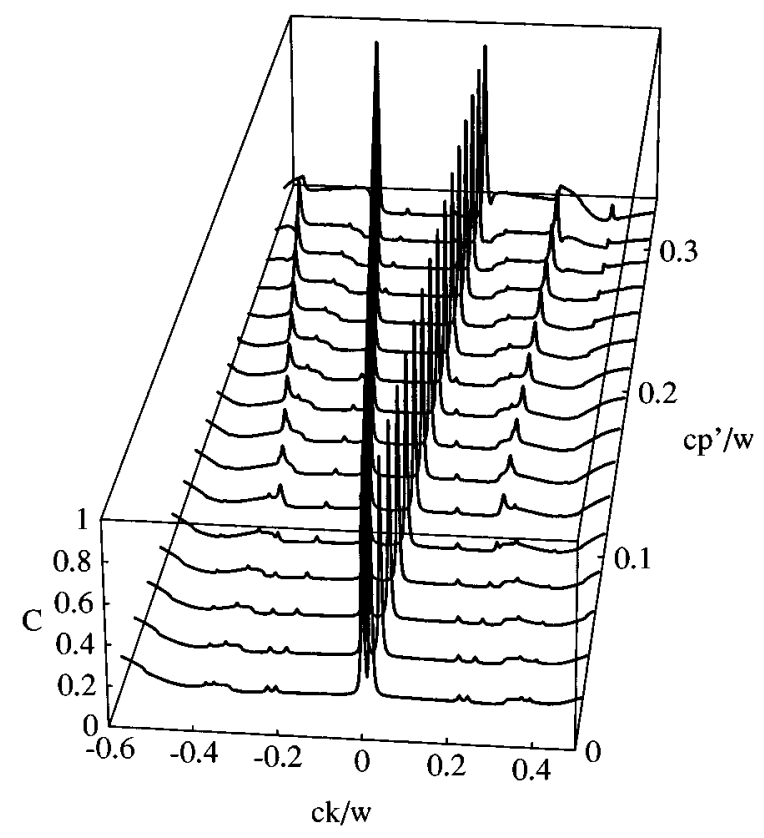

FIG. 4. Same as Fig. 2 but for $p$ polarization.

first two combinations give rise to the eight degenerate ME peaks that are observed therein. Note that these peaks are weaker than those for $s$ polarization, as expected from the fact that the roughness-induced light coupling into TE GWs is stronger than that into TM modes.

It should be remarked that the results presented thus far have been obtained for a random rough surface with a RPS that enhances the excitation of GWs and suppresses the lowest-order contribution to the intensity angular correlations. In this manner, the predicted degenerate ME peaks, originated in the double (or higher) scattering contributions, have been neatly reproduced in the perturbation-theoretic calculations previously shown, despite the smoothness (small $\delta / \lambda)$ of the surface. Thus it would be interesting to show whether these peaks can be seen when a strong single scattering contribution is present.

To this end, the intensity angular correlation [Eq. (2.21)] is now calculated for a rough surface with a GPS (3.1). The resulting ME envelopes are shown in Figs. 5 and 6 for $s$ and $p$ polarization, respectively, with the same conventions as in Figs. 2 and 4. Unlike in Figs. 2 and 4 for the RPS, in Figs. 5 and 6 (GPS) the expected degenerate ME peaks [cf. Eqs. (3.5)] and the $C=1$ maxima [cf. Eqs. (3.4)] rise over a high correlation background which is basically due to the secondorder single scattering contribution $C_{E, \gamma}^{(1-1)}$, Eq. (A1). This is corroborated in Fig. 7, where we plot the contributions to the $s$-polarized ME envelope (see Fig. 5 for $c p^{\prime} / \omega=0.1$ ) coming from the terms in the amplitude correlation of second order, $C_{E, s}^{(1-1)}(\mathrm{A} 1)$, and of fourth order: ladder $C_{E, s}^{(2-2) L}$ (A2), crossed $C_{E, s}^{(2-2) C}(\mathrm{~A} 3)$, and (1-3) $C_{E, s}^{(1-3)}(\mathrm{A} 4)$. Note that, as in Fig. 3, the interference terms resulting from the square modulus in Eq. (2.21) have not been included; thus the sum of all the contributions in Figs. 3 and 7 needn't be equal to $C_{s}^{\mathrm{ME} \text {,diff }}$. In Fig. 7 it is clearly seen that, as expected, the maximum at $k=k^{\prime}$ and the corresponding degenerate ME peaks [cf. Eqs. (3.5a)] are given by the ladder contribution, whereas the maximally crossed contribution accounts for 


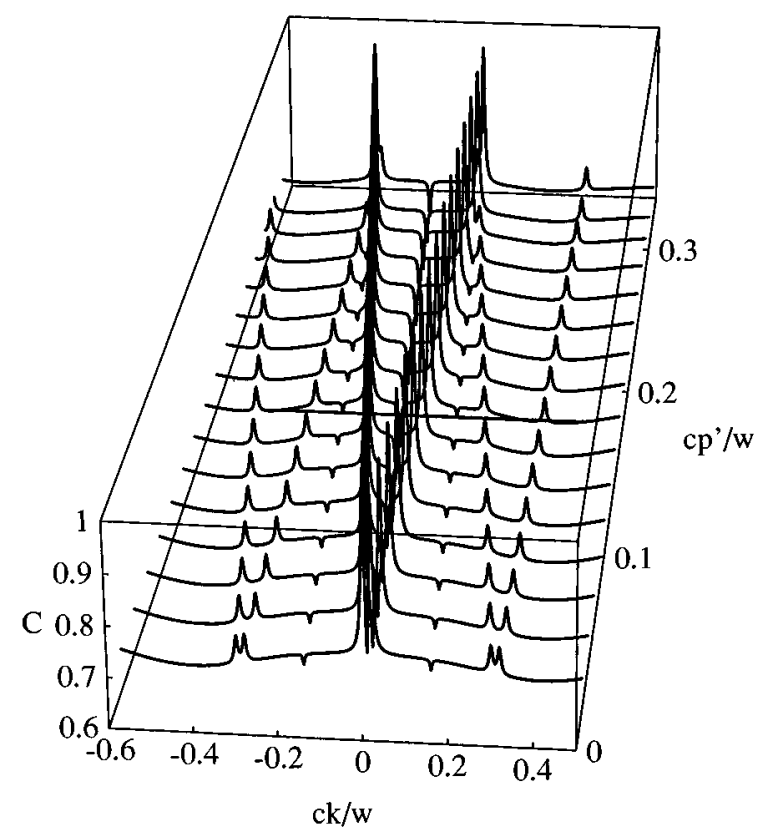

FIG. 5. Same as Fig. 2 but for a random rough surface with a Gaussian power spectrum [cf. Eq. (3.1)] with $a=100 \mathrm{~nm}$.

those peaks following the conditions (3.4b) and (3.5b). Interestingly enough, in spite of the strong single scattering contribution as compared to the double scattering one (as required for the validity of the perturbation theory), the various peaks appearing in the latter are still easily distinguishable from the structure-less background. Also in contrast to the results for the RPS (see Figs. 2 and 4), with the use of the GPS, which is a smooth function of the wave vector, none of the degenerate ME peaks exhibit a sharp cutoff for certain value of $p^{\prime}$ (see Figs. 5 and 6). On the other hand, the small dips observed in Figs. 5, 6, and 7 result from the normalization of the correlation function by the mean scattered inten-

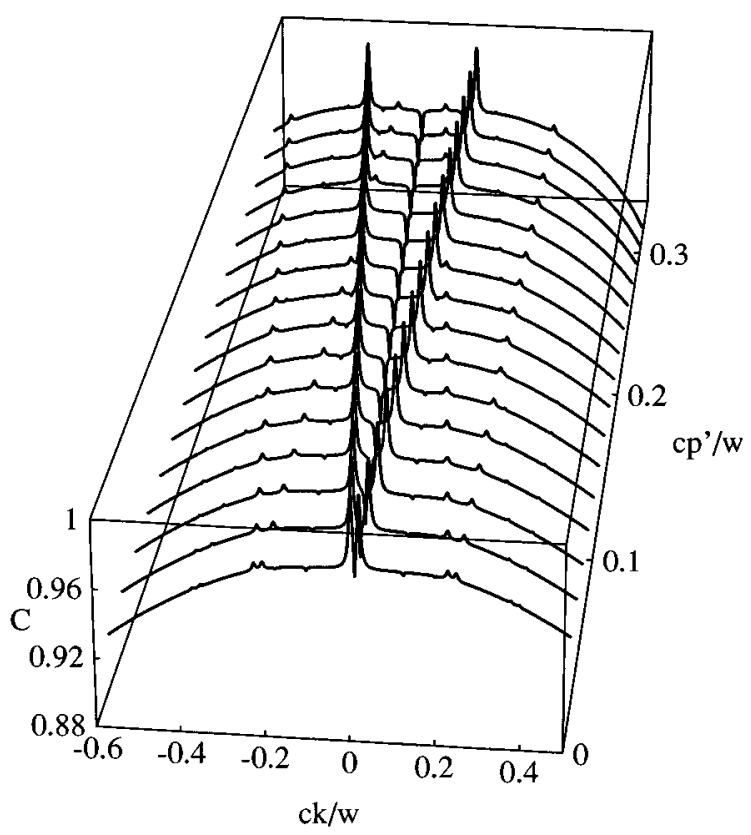

FIG. 6. Same as Fig. 5 but for $p$ polarization.

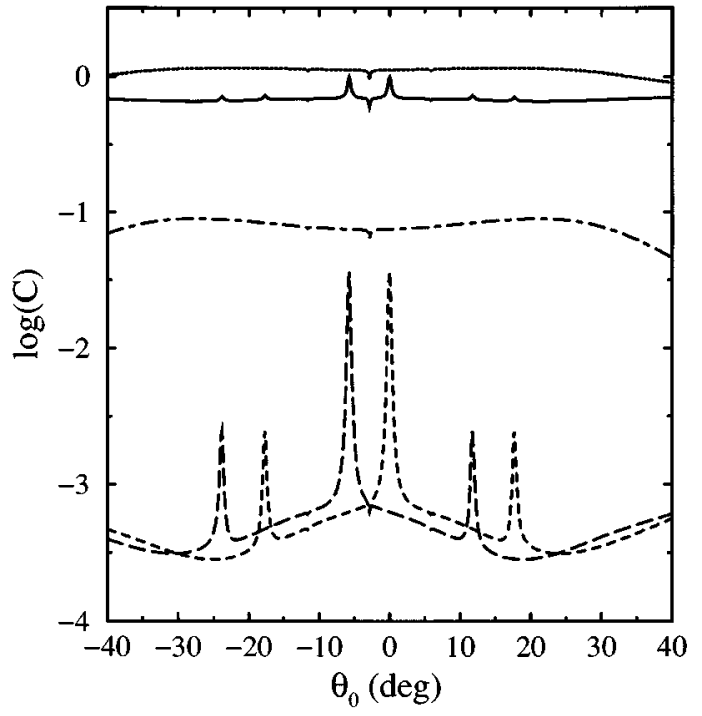

FIG. 7. Same as Fig. 5 but only for $c p^{\prime} / \omega=0.1$. The incident angle along the $x$ axis is $\theta_{0}=\sin ^{-1}(c k / \omega)$. The second-order contribution (dotted curve), and the fourth-order contributions arising from the ladder (dashed curve), crossed (long dashed curve), the (1-3) (dash-dot curve) terms are shown separately, as well as the total $C$ (solid curve), in a logarithmic scale.

sity, which displays degenerate backscattering, satellite peaks precisely at such positions. ${ }^{15,17}$

It should be mentioned that the ME peak and its timereversed partner [Eqs. (3.4)] shown in Figs. 2-7 have the same origin as the degenerate ME peaks, but require that the same GW be involved in the double scattering paths undergoing interference [cf. Eqs. (3.5) for $q_{1}=q_{2}$ ]. (Consequently, only one GW polariton is needed for them to appear.) Strictly speaking, these peaks are only fourth-order corrections in the amplitude correlation function to the entire ME. In the case of the RPS, it has been demonstrated that the second-order term vanishes. Nonetheless, this does not occur for the GPS, for which a strong second-order contribution is present (see Figs. 5, 6, and 7). This second order, single scattering contribution yields, in fact, a large memory effect centered at the positions (3.4) that decays so slowly with $k$ that the decrease is hardly appreciable within the angular range of Figs. 5, 6, and 7. This behavior manifests the strong speckle correlation, even for large angular separations, in the light scattered from very smooth surfaces for which the single scattering contribution predominates; it was reported for perfectly conducting, slowly varying surfaces in Ref. 3 long before the term "memory effect" was coined. ${ }^{5}$

\section{CONCLUSIONS}

In this paper, we have found alternative features in the angular dependence of the intensity correlation function $C\left(p, k ; p^{\prime}, k^{\prime}\right)$ of the scattered electromagnetic waves in a system consisting of a dielectric film with a one-dimensional, randomly rough surface deposited on a planar, perfectly conducting substrate. To study the angular intensity correlation, a perturbation theory of the angular amplitude correlation through terms up to fourth order in the surface profile function $\zeta\left(x_{1}\right)$ has been developed for both $s$ and $p$ polarization 
[cf. Eqs. (2.20), (2.21), and (A1)-(A4)]. This amplitude correlation, whose square modulus is the intensity correlation when the factorization approximation is invoked, is nonzero only for $p-k=p^{\prime}-k^{\prime}$ (also known as ME condition). The mean thickness of the film must be such that, in the absence of roughness, it supports at least two degenerate GW polaritons which have different values of their wave numbers at a given frequency of the incoming beam. Two different power spectra of the rough surface have been considered: a rectangle (RPS) centered about the guided wave numbers that enhances the light coupling for near normal incidence, and a slowly varying (small correlation length) Gaussian (GPS).

Under these circumstances, for each possible combination of two GWs, we observe two satellite peaks symmetrically placed about the ME maximum, and other two satellite peaks symmetrically placed about the time-reversed ME maximum. The positions of these peaks are predicted by Eqs. (3.5a) and (3.5b), respectively, which result from a simple argument based on the phase coherence condition for multiply scattered trajectories mediated by degenerate $\mathrm{GW}$ polaritons. This phenomenon is referred to as degenerate memory effect: It has been confirmed by our perturbation-theoretic calculations for both $s$ polarization, in the case that the dielectric film supports two TE GWs for the choice of scattering parameters, and for $p$ polarization, supporting three TM GW for the same parameters. The degenerate ME peaks are larger for $s$ polarization than those for $p$ polarization, as expected from the fact that the roughness-induced coupling into guided waves is stronger for the former. The same argument given above, but involving only one GW, explains the existence of similar peaks as a multiple scattering contribution to the intensity correlation function at the positions of the above mentioned ME maximum and its time reversed partner [cf. Eqs. (3.4a) and (3.4b), respectively]. The analysis of the vari- ous contributions through terms of fourth order to the amplitude correlation function reveals that those peaks at the positions (3.4a) and (3.5a) stem from the ladder term, whereas the maximally crossed term yields those at the positions (3.4b) and (3.5b).

The degenerate ME is enhanced for the rough surface with the RPS, as are all the double scattering effects, since no second-order, single scattering contribution is present. On the other hand, for the rough surface with the GPS, the degenerate ME peaks are less clearly observed, for they appear superimposed to the strong single scattering background, which in turn accounts for the weakly decaying ME expected for such a smooth rough surface. From the experimental point of view, it would be much simpler to observe the degenerate $\mathrm{ME}$ by the use of a one-dimensionally-rough dielectric film with the appropriate RPS, as has been done in Ref. 19 for the study of the backscattering peak from rough metal surfaces involving the excitation of surface-plasmon polaritons. In the case of the GPS with a small correlation length (in terms of the wavelength), the degenerate ME could be observed more easily for $s$-polarized electromagnetic waves.

\section{ACKNOWLEDGMENTS}

This work was supported by the Consejo Superior de Investigaciones Científicas and the Dirección General de Investigación Científica y Técnica through Grant No. PB930973-C02-02.

\section{APPENDIX}

The contributions to the amplitude correlation function through terms of fourth order in the surface profile function, Eq. (2.21), can be written in the form

$$
\begin{gathered}
C_{E, \gamma}^{(1-1)}\left(p, k ; p^{\prime}, k^{\prime}\right)=L \delta^{2} g(|p-k|) v_{\gamma}^{(1)}(p \mid k)\left[v_{\gamma}^{(1)}\left(p^{\prime} \mid k^{\prime}\right)\right]^{*}, \\
C_{E, \gamma}^{(2-2) L}\left(p, k ; p^{\prime}, k^{\prime}\right)=L \delta^{4} \int_{-\infty}^{\infty} \frac{d q}{2 \pi} g(|p-q|) g(|q-k|) \chi_{\gamma}^{(2-2)}\left(p|q| k ; p^{\prime}\left|q-k+k^{\prime}\right| k^{\prime}\right), \\
C_{E, \gamma}^{(2-2) C}\left(p, k ; p^{\prime}, k^{\prime}\right)=L \delta^{4} \int_{-\infty}^{\infty} \frac{d q}{2 \pi} g(|p-q|) g(|q-k|) \chi_{\gamma}^{(2-2)}\left(p|q| k ; p^{\prime}\left|k-q+p^{\prime}\right| k^{\prime}\right), \\
C_{E, \gamma}^{(1-3)}\left(p, k ; p^{\prime}, k^{\prime}\right)=L \delta^{4}\left[\Psi \Psi_{\gamma}\left(p, k ; p^{\prime}, k^{\prime}\right)+\Psi_{\gamma}^{*}\left(p^{\prime}, k^{\prime} ; p, k\right)\right],
\end{gathered}
$$

where

$$
\begin{aligned}
\Psi_{\gamma}\left(p, k ; p^{\prime}, k^{\prime}\right)= & g\left(\left|p^{\prime}-k^{\prime}\right|\right)\left[v_{\gamma}^{(1)}\left(p^{\prime} \mid k^{\prime}\right)\right]^{*} \int_{-\infty}^{\infty} \frac{d q}{2 \pi}\left\{g(|p-q|) \chi_{\gamma}^{(1-3)}(p|q| p \mid k)+g(|q-k|)\left[\chi_{\gamma}^{(1-3)}(p|k| q \mid k)\right.\right. \\
& \left.\left.+\chi_{\gamma}^{(1-3)}(p|p-k+q| q \mid k)\right]\right\}
\end{aligned}
$$

the functions $\chi_{\gamma}^{(2-2)}$ and $\chi_{\gamma}^{(1-3)}$ in the integrals of Eqs. (A2) and (A3), and Eq. (A5), respectively, are given by

$$
\begin{aligned}
\chi^{(2-2)}\left(p|q| k ; p^{\prime}\left|q^{\prime}\right| k^{\prime}\right)= & v_{\gamma}^{(2)}(p|q| k)\left[v_{\gamma}^{(2)}\left(p^{\prime}\left|q^{\prime}\right| k^{\prime}\right)\right]^{*}+v^{(2)}(p|q| k)\left[v_{\gamma}^{(1)}\left(p^{\prime} \mid q^{\prime}\right) G_{0}\left(q^{\prime}, \omega\right) v_{\gamma}^{(1)}\left(q^{\prime} \mid k^{\prime}\right)\right]^{*} \\
& +v_{\gamma}^{(1)}(p \mid q) G_{0}(q, \omega) v_{\gamma}^{(1)}(q \mid k)\left[v^{(2)}\left(p^{\prime}\left|q^{\prime}\right| k^{\prime}\right)\right]^{*}+v_{\gamma}^{(1)}(p \mid q) G_{0}(q, \omega) v_{\gamma}^{(1)}(q \mid k) \\
& \times\left[v_{\gamma}^{(1)}\left(p^{\prime} \mid q^{\prime}\right) G_{0}\left(q^{\prime}, \omega\right) v_{\gamma}^{(1)}\left(q^{\prime} \mid k^{\prime}\right)\right]^{*}
\end{aligned}
$$




$$
\begin{aligned}
\chi_{\gamma}^{(1-3)}\left(p|q| q^{\prime} \mid k\right)= & v_{\gamma}^{(3)}\left(p|q| q^{\prime} \mid k\right)+v_{\gamma}^{(2)}\left(p|q| q^{\prime}\right) G_{0}\left(q^{\prime}, \omega\right) v_{\gamma}^{(1)}\left(q^{\prime} \mid k\right)+v_{\gamma}^{(1)}(p \mid q) G_{0}(q, \omega) v_{\gamma}^{(2)}\left(q\left|q^{\prime}\right| k\right) \\
& +v_{\gamma}^{(1)}(p \mid q) G_{0}(q, \omega) v_{\gamma}^{(1)}\left(q \mid q^{\prime}\right) G_{0}\left(q^{\prime}, \omega\right) v_{\gamma}^{(1)}\left(q^{\prime} \mid k\right)
\end{aligned}
$$

The functions $v_{\gamma}^{(1)}, v_{\gamma}^{(2)}$, and $v_{\gamma}^{(3)}$, as mentioned in the text, have been calculated in Ref. 15 [cf. Eqs. (A14)] for $s$ polarization, and in Ref. 17 [cf. Eqs. (A1)-(A4)] for $p$ polarization.

${ }^{1}$ Scattering and Localization of Classical Waves in Random Media, edited by P. Sheng (World Scientific, Singapore, 1990).

${ }^{2}$ Scattering in Volumes and Surfaces, edited by M. NietoVesperinas and J. C. Dainty (North-Holland, Amsterdam, 1990).

${ }^{3}$ D. Léger, E. Mathieu, and J. C. Perrin, Appl. Opt. 14, 872 (1975); D. Léger and J. C. Perrin, J. Opt. Soc. Am. 66, 1210 (1976).

${ }^{4}$ J. W. Goodman, in Laser Speckle and Related Phenomena, edited by J. C. Dainty (Springer-Verlag, Berlin, 1984).

${ }^{5}$ S. Feng, C. Kane, P. A. Lee, and A. D. Stone, Phys. Rev. Lett. 61, 834 (1988).

${ }^{6}$ I. Freund, M. Rosenbluh, and S. Feng, Phys. Rev. Lett. 61, 2328 (1988)

${ }^{7}$ R. Berkovits, M. Kaveh, and S. Feng, Phys. Rev. B 40, 737 (1989).

${ }^{8}$ R. Berkovits and M. Kaveh, Phys. Rev. B 41, 2635 (1990).

${ }^{9}$ R. Berkovits and M. Kaveh, Europhys. Lett. 13, 97 (1990).

${ }^{10}$ T. R. Michel and K. A. O’Donnell, J. Opt. Soc. Am. A 9, 1374 (1992).

${ }^{11}$ M. Nieto-Vesperinas and J. A. Sánchez-Gil, J. Opt. Soc. Am. A 10, 150 (1993).

${ }^{12}$ M. Nieto-Vesperinas and J. A. Sánchez-Gil, Phys. Rev. B 46, 3112 (1992); 48, 4132 (1993).
${ }^{13}$ H. M. Escamilla, E. R. Méndez, and D. F. Hotz, Appl. Opt. 32, 2734 (1993).

${ }^{14}$ V. D. Freilikher, M. Pustilnik, and I. Yurkevich, Phys. Lett. A 193, 467 (1994).

${ }^{15}$ J. A. Sánchez-Gil, A. A. Maradudin, J. Q. Lu, V. D. Freilikher, M. Pustilnik, and I. Yurkevich, Phys. Rev. B 50, 15353 (1994).

${ }^{16}$ J. A. Sánchez-Gil, A. A. Maradudin, J. Q. Lu, and V. D. Freilikher, Phys. Rev. B 51, 17100 (1995).

${ }^{17}$ J. A. Sánchez-Gil, A. A. Maradudin, J. Q. Lu, V. D. Freilikher, M. Pustilnik, and I. Yurkevich, J. Mod. Opt. 43, 435 (1996).

${ }^{18}$ J. Q. Lu, J. A. Sánchez-Gil, E. R. Méndez, Z.-H. Gu, and A. A. Maradudin (unpublished).

${ }^{19}$ C. S. West and K. A. O'Donnell, J. Opt. Soc. Am. A 12, 390 (1995); Opt. Lett. 21, 1 (1996).

${ }^{20}$ A. A. Maradudin, A. R. McGurn, and E. R. Méndez, J. Opt. Soc. Am. A 12, 2500 (1995).

${ }^{21}$ G. C. Brown, V. Celli, M. Coopersmith, and M. Haller, Surf. Sci. 129, 507 (1983).

${ }^{22}$ J. C. Dainty, J. Opt. Soc. Am. 62, 595 (1972).

${ }^{23}$ J. Ohtsubo and T. Asakura, Opt. Lett. 1, 98 (1977); J. Ohtsubo, Appl. Opt. 27, 1290 (1988).

${ }^{24}$ A. Arsenieva and S. Feng, Phys. Rev. B 47, 13047 (1993). 